\title{
INTERMEDIATE REGULARITY RESULTS FOR THE SOLUTION OF A HIGH ORDER PARABOLIC EQUATION
}

\author{
AREZKI KHELOUFI
}

Received 19 September, 2019

\begin{abstract}
In this work we give new intermediate regularity results for the solution of the follow-
\end{abstract} ing $2 m$-th order parabolic equation

$$
\partial_{t} u+(-1)^{m} \sum_{i=1}^{n} \partial_{x_{i}}^{2 m} u=0,
$$

where $m$ is a positive integer, subject to Dirichlet condition on the lateral boundary of a cylindrical domain and to a non-homogeneous initial Cauchy data.

2010 Mathematics Subject Classification: 35K05; 35K55

Keywords: regularity results, parabolic equations, Sobolev spaces

\section{INTRODUCTION}

Let $\Omega$ be an open bounded set of $\mathbb{R}^{n}$ with boundary $\Gamma$ and $Q$ the cylinder $\mathbb{R}_{+} \times \Omega$ with lateral boundary $\Sigma=\mathbb{R}_{+} \times \Gamma$. We assume that $\Omega$ is of class $C^{2 m}$. Consider in $Q$ the following boundary value problem:

$$
\left\{\begin{array}{l}
\partial_{t} u+(-1)^{m} \sum_{i=1}^{n} \partial_{x_{i}}^{2 m} u=0 \text { in } Q, \\
\partial_{v}^{j} u=0 \text { on } \Sigma, j=0,1, \ldots, m-1, \\
u(0, x)=u_{0}(x), x \in \Omega
\end{array}\right.
$$

where $m$ is a positive integer and $\partial_{v}^{j}$ is the derivative of order $j$ throughout the normal vector $v$ on $\Sigma$.

Classical results on the resolution of Problem (1.1) when the initial Cauchy data $u_{0}$ belongs to $L^{2}(\Omega)$ or to the usual Sobolev space $H_{0}^{m}(\Omega)$ can be found in [6], see also [1], [2], [8] and [9] and the references therein.

Our interest in this work is the regularity of the solution $u$ of (1.1) in terms of the regularity of the initial data $u_{0}$. More precisely, we are interested on the question of the regularity of the solution of (1.1) when $u_{0}$ is "between" $H_{0}^{m}(\Omega)$ and $L^{2}(\Omega)$. The 
results obtained here complement those obtained for the heat equation, i.e. $m=1$, in [4].

The organization of this paper is as follows. In Section 2, we begin by preliminaries where we define the basic functional spaces, in which we will work and we give some of their properties needed for our study. Then, we recall a classical result for Problem (1.1) and we prove a fundamental lemma which will allow us to prove our main result in Section 3.

\section{PRELIMINARIES}

\subsection{Function spaces}

In this subsection, we recall the definitions of the basic functional spaces, in which we will work. We will need some anisotropic Sobolev spaces (see [6]), which we recall in the following definitions

$$
H^{r, s}\left(\mathbb{R}^{n}\right)=\left\{u \in L^{2}\left(\mathbb{R}^{n}\right):\left[\left(1+\zeta^{2}\right)^{r / 2}+\left(1+\tau^{2}\right)^{s / 2}\right] \widehat{u} \in L^{2}\left(\mathbb{R}^{n}\right)\right\}
$$

where $\widehat{u}$ is the Fourier transform of $u$ and $r, s$ are two non-negative numbers. We put

$$
H^{r, s}(\Omega)=\left\{\left.u\right|_{\Omega}: u \in H^{r, s}\left(\mathbb{R}^{n}\right)\right\},
$$

with $\Omega$ is an open subset of $\mathbb{R}^{n}$. For $0 \leq r \leq 1$ and a positive integer $m$, we recall that the space $H^{r, 2 m r}(Q)$, where $Q=\mathbb{R}_{+} \times \Omega$, can be defined by

$$
H^{r, 2 m r}(Q)=L^{2}\left(\mathbb{R}_{+}, H^{2 m r}(\Omega)\right) \cap H^{r}\left(\mathbb{R}_{+}, L^{2}(\Omega)\right)
$$

and the space $H_{0}^{r}(\Omega)$ is defined by

$$
H_{0}^{r}(\Omega)=\left\{u \in H^{r}(\Omega) ; u=0 \text { on } \Gamma\right\}
$$

for $\frac{1}{2}<r \leq 1, \Gamma$ is the boundary of $\Omega$.

$$
H_{0}^{r}(\Omega)=H_{00}^{\frac{1}{2}}(\Omega)
$$

for $r=\frac{1}{2}$,

for $0 \leq r<\frac{1}{2}$.

$$
H_{0}^{r}(\Omega)=H^{r}(\Omega)
$$

We will need also some interpolation spaces in Hilbert spaces (see [7] and [3]), which we recall in the following definition.

Let $X, Y$ be two Hilbert spaces with

$$
X \subset Y \text { continuously. }
$$

Here, we give one of the usual methods, namely, that of Lions-Peetre [7] which allow us to build spaces

$$
[X, Y]_{\theta} 0<\theta<1,
$$

"intermediate" between $X$ and $Y$. 
Definition 1. The space $[X, Y]_{\theta} 0<\theta<1$, is a sub-space of $Y$ consisting of elements $a$ which can be written in the form

$$
a=\int_{0}^{\infty} u(t) \frac{d t}{t}
$$

with

$$
t^{\theta} u(t) \in L_{*}^{2}(X), t^{\theta-1} u(t) \in L_{*}^{2}(Y) .
$$

This space is endowed with the norm

$$
a \longmapsto \inf \left[\left(\int_{0}^{\infty} t^{2 \theta}|u(t)|_{X}^{2} \frac{d t}{t}\right)^{\frac{1}{2}}+\left(\int_{0}^{\infty} t^{2(\theta-1)}|u(t)|_{Y}^{2} \frac{d t}{t}\right)^{\frac{1}{2}}\right] ;
$$

the inf is taken with respect to $u$ verifying (2.1) and (2.2).

Here $L_{*}^{2}(V)$ denotes the space of functions from $t>0$ with values in $V$, which are square integrable for the Haar measure $d t / t$.

Example 1. $H^{r, s}(\Omega)$ can also be defined as a real interpolation space between $H^{r /(1-\theta), s /(1-\theta)}(\Omega)$ and $\left.L^{2}(\Omega), \theta \in\right] 0,1[$, (see [10])

$$
H^{r, s}(\Omega)=\left[H^{r /(1-\theta), s /(1-\theta)}(\Omega), L^{2}(\Omega)\right]_{\theta} .
$$

In this work, we consider the case $s=2 m r, \theta=1-r$,

$$
\left.H^{r, 2 m r}(\Omega)=\left[H^{1,2 m}(\Omega), L^{2}(\Omega)\right]_{1-r} \quad \forall r \in\right] 0,1[.
$$

The space $H^{r, 2 m r}(\Omega)$ is well defined by Relationship (2.4) because the right hand side term of (2.4) is well defined as an interpolation space between two well defined spaces $H^{1,2 m}(\Omega)$ and $L^{2}(\Omega)$.

Example 2. The usual Sobolev spaces $H^{s}(\Omega)(s \geq 0)$ can be defined by interpolation

$\theta \in] 0,1[$.

$$
H^{s}(\Omega)=\left[H^{s /(1-\theta)}(\Omega), L^{2}(\Omega)\right]_{\theta},
$$

Hereafter some interpolation theory properties on spaces $[.,]_{\theta}$ (see Triebel [10]), needed for proving our main result in the following section.

Theorem 1 ([10]). Let $A_{0}, A_{1}$ be two Hilbert spaces with

$$
A_{0} \subset A_{1} \text { continuously. }
$$

Then

$0<\theta<1$.

$$
\left[L^{2}\left(A_{0}\right), L^{2}\left(A_{1}\right)\right]_{\theta}=L^{2}\left(\left[A_{0}, A_{1}\right]_{\theta}\right),
$$

A direct consequence of Theorem 1 is 
Corollary 1. For each $0 \leq r \leq 1$ and any positive integer $m$, we have

$$
\begin{aligned}
{\left[L^{2}\left(\mathbb{R}_{+}, H^{2 m}(\Omega)\right), L^{2}\left(\mathbb{R}_{+}, H^{m}(\Omega)\right)\right]_{1-r} } & =L^{2}\left(\mathbb{R}_{+},\left[H^{2 m}(\Omega), H^{m}(\Omega)\right]_{1-r}\right) \\
& =L^{2}\left(\mathbb{R}_{+}, H^{m(1+r)}(\Omega)\right) .
\end{aligned}
$$

with $\Omega$ an open bounded set of $\mathbb{R}^{n}$.

\subsection{Lemmas}

The next result is well known ( cf. Lions and Magenes [6]).

Lemma 1. 1) For given $u_{0}$ in $H_{0}^{m}(\Omega)$, Problem (1.1) has a unique solution $u$ in $H^{1,2 m}(Q)$ defined by

$$
H^{1,2 m}(Q)=L^{2}\left(\mathbb{R}_{+}, H^{2 m}(\Omega)\right) \cap H^{1}\left(\mathbb{R}_{+}, L^{2}(\Omega)\right) .
$$

2) For given $u_{0}$ in $L^{2}(\Omega)$, Problem (1.1) has a unique weak solution $u$ in

$$
L^{2}\left(\mathbb{R}_{+}, H_{0}^{m}(\Omega)\right) \cap H^{1}\left(\mathbb{R}_{+}, H^{-m}(\Omega)\right) \cap L^{\infty}\left(\mathbb{R}_{+}, L^{2}(\Omega)\right) .
$$

We will need the following lemma for proving our main result in the following section.

Lemma 2. Let $u_{0} \in L^{2}(\Omega)$. Then the solution $u$ of Problem (1.1) associated to $u_{0}$ is in $H^{\frac{1}{2}}\left(\mathbb{R}_{+}, L^{2}(\Omega)\right)$. Moreover, there exists a positive constant $C$ (independent of $\left.u_{0}\right)$ such that

$$
\|u\|_{H^{\frac{1}{2}}\left(\mathbb{R}_{+}, L^{2}(\Omega)\right)} \leq C\left\|u_{0}\right\|_{L^{2}(\Omega)} .
$$

Proof. Consider a sequence of spectral elements $\left(\lambda_{k}, \varphi_{k}\right), k \in \mathbb{N}$ of the Dirichlet problem for the operator $(-1)^{m} \sum_{i=1}^{n} \partial_{x_{i}}^{2 m}$

$$
\left\{\begin{array}{l}
(-1)^{m} \sum_{i=1}^{n} \partial_{x_{i}}^{2 m} \varphi_{k}=\lambda_{k} \varphi_{k} \\
\varphi_{k} \in H_{0}^{m}(\Omega) \\
\left\|\varphi_{k}\right\|_{L^{2}(\Omega)}=1 .
\end{array}\right.
$$

The sequence $\left(\varphi_{k}\right)_{k \in \mathbb{N}}$ is a basis of $L^{2}(\Omega)$. If $u_{0} \in L^{2}(\Omega)$ we may write

$$
u_{0}(x)=\sum_{k \in \mathbb{N}} a_{k} \varphi_{k}(x)
$$

with $\left\|u_{0}\right\|_{L^{2}(\Omega)}^{2}=\sum_{k \in \mathbb{N}} a_{k}^{2}$. The solution associated to $u_{0}$ is

$$
u(t, x)=\sum_{k \in \mathbb{N}} a_{k} \exp \left(-\lambda_{k} t\right) \varphi_{k}(x) .
$$

Note $\widetilde{u}$ the extension of $u$ to $\mathbb{R}$, i.e.,

$$
\widetilde{u}(t, x)=\sum a_{k} \exp \left|-\lambda_{k} t\right| \varphi_{k}(x) .
$$


By the Fourier transform

$$
\widehat{u}(\zeta, x)=C \sum \frac{a_{k} \lambda_{k}}{\zeta^{2}+\lambda_{k}^{2}} \varphi_{k}(x),
$$

where $C$ is a constant, from which

$$
\|\widehat{u}(\zeta, .)\|^{2}=C^{2} \sum a_{k}^{2} \frac{\lambda_{k}^{2}}{\left(\zeta^{2}+\lambda_{k}^{2}\right)^{2}}
$$

and by elementary calculations, we check easily that

$$
\int_{\mathbb{R}}|\zeta|\|\widehat{\widetilde{u}}(\zeta, .)\|^{2} d \zeta=C^{\prime} \sum a_{k}^{2}
$$

where $C^{\prime}$ is a constant. Consequently

$$
\widetilde{u} \in H^{\frac{1}{2}}\left(\mathbb{R}, L^{2}(\Omega)\right),
$$

then

$$
u \in H^{\frac{1}{2}}\left(\mathbb{R}_{+}, L^{2}(\Omega)\right)
$$

by restriction of $\widetilde{u}$ to $t>0$.

\section{MAIN RESULT}

In the sequel, we will assume that $u_{0} \in H_{0}^{r m}(\Omega), 0 \leq r \leq 1$. Thus $H_{0}^{r m}(\Omega)$ is the interpolation space of order $1-r$ between $H_{0}^{m}(\Omega)$ and $L^{2}(\Omega)$. Indeed, it suffices to take $\theta=1-r$ and $s=r m$ in Example 2. We look for the regularity of $u$ in term of $r$. Our main result in this work is

Theorem 2. For given $u_{0}$ in $H_{0}^{r m}(\Omega) 0 \leq r \leq 1$, Problem (1.1) has a unique weak solution $u$ in $H^{\frac{1+r}{2}, m(1+r)}(Q)$.

Proof. Let $u_{0} \in H_{0}^{r m}(\Omega) 0 \leq r \leq 1$ then $u_{0} \in L^{2}(\Omega)$ and consequently (1.1) admits a unique weak solution (see Lemma 1$) u$ in $L^{2}\left(\mathbb{R}_{+}, H_{0}^{m}(\Omega)\right)$. In order to show that this solution is in $L^{2}\left(\mathbb{R}_{+}, H^{m(1+r)}(\Omega)\right)$ it suffices to interpolate the operator $S$ which associates $u$ to $u_{0}$. Indeed $S: u_{0} \mapsto u$ is linear continuous from $H_{0}^{m}(\Omega)$ to $L^{2}\left(\mathbb{R}_{+}, H^{2 m}(\Omega)\right)$ and from $L^{2}(\Omega)$ to $L^{2}\left(\mathbb{R}_{+}, H^{m}(\Omega)\right)$. By interpolation, it is linear continuous from

$$
\left[H_{0}^{m}(\Omega), L^{2}(\Omega)\right]_{1-r} \text { into }\left[L^{2}\left(\mathbb{R}_{+}, H^{2 m}(\Omega)\right), L^{2}\left(\mathbb{R}_{+}, H^{m}(\Omega)\right)\right]_{1-r} .
$$

Thanks to Corollary $1, S$ is linear continuous from

$$
H_{0}^{r m}(\Omega) \text { into } L^{2}\left(\mathbb{R}_{+}, H^{m(1+r)}(\Omega)\right) .
$$


We can interpolate again $S$ for proving that $u \in H^{\frac{r+1}{2}}\left(\mathbb{R}_{+}, L^{2}(\Omega)\right)$. Indeed, $S$ is linear continuous from $L^{2}(\Omega)$ into $H^{\frac{1}{2}}\left(\mathbb{R}_{+}, L^{2}(\Omega)\right)$ (see Lemma 2) and from $H_{0}^{m}(\Omega)$ into $H^{1}\left(\mathbb{R}_{+}, L^{2}(\Omega)\right)$ (see Theorem 1). By interpolation, it is linear continuous from

$$
\left[H_{0}^{m}(\Omega), L^{2}(\Omega)\right]_{1-r} \text { into }\left[H^{\frac{1}{2}}\left(\mathbb{R}_{+}, L^{2}(\Omega)\right), H^{1}\left(\mathbb{R}_{+}, L^{2}(\Omega)\right)\right]_{1-r} .
$$

But, (see Triebel [10])

$$
\left[H^{\frac{1}{2}}\left(\mathbb{R}_{+}, L^{2}(\Omega)\right), H^{1}\left(\mathbb{R}_{+}, L^{2}(\Omega)\right)\right]_{1-r}=H^{\frac{r+1}{2}}\left(\mathbb{R}_{+}, L^{2}(\Omega)\right) .
$$

Then, $S$ is linear continuous from $H_{0}^{r m}(\Omega)$ into $H^{\frac{r+1}{2}}\left(\mathbb{R}_{+}, L^{2}(\Omega)\right)$. This ends the proof of Theorem 2.

Remark 1. Note that the anisotropic Hilbert spaces used in this work may be extended to the case of parabolic Sobolev spaces built on $L^{p}, p \neq 2$. An idea for this extension can be found in [5].

\section{ACKNOWLEDGEMENT}

The author is grateful to anonymous reviewers for carefully reading this paper and for their valuable comments and suggestions, which have improved the paper.

\section{REFERENCES}

[1] S. Gala, Q. Liu, and M. A. Ragusa, "Logarithmically improved regularity criterion for the nematic liquid crystal flows in $B_{\infty, \infty}^{-1}$ space." Computers and Mathematics with Applications, vol. 65, pp. 1738-1745, 2013, doi: 10.1016/j.camwa.2013.04.003.

[2] V. A. Galaktionov, "On regularity of a boundary point for higher-order parabolic equations: towards Petrovskii-type criterion by blow-up approach.” Nonlinear Differ. Equ. Appl., vol. 16, no. 5, pp. 597-655, 2009, doi: 10.1007/s00030-009-0025-x.

[3] P. Grisvard, "P. Grisvard, Caractérisation de quelques espaces d'interpolation." Arch. Rational Mech. Anal., vol. 25, no. 1, pp. 40-63, 1967, doi: 10.1007/BF00281421.

[4] A. Kheloufi and B. Sadallah, "On the regularity of the heat equation solution: Two approaches." Applied Mathematics and Computation, vol. 218, no. 5, pp. 1623-1633, 2011, doi: 10.1016/j.amc.2011.06.042.

[5] J. L. Lions, "Quelques remarques sur les problèmes de Dirichlet et de Neumann." Séminaire Jean Leray, no. 6, pp. 1-18, 1961-1962.

[6] J. L. Lions and E. Magenes, Problèmes aux Limites Non Homogènes et Applications 1, 2. Paris: Dunod, 1968.

[7] J. L. Lions and J. Peetre, "Sur une classe d'espaces d'interpolation." Publ. Math. de l'I. H. E. S., no. 19, pp. 5-68, 1964, doi: 10.1007/BF02684796.

[8] M. A. Ragusa, "Cauchy-Dirichlet problem associated to divergence form parabolic equations." Communications in Contemporary Mathematics, vol. 6, no. 3, pp. 377-393, 2004.

[9] B. Sadallah, "Spectral properties of even order derivative operators." Appl. Math. E-Notes, pp. 10-15, 2003.

[10] H. Triebel, Interpolation Theory, Function Spaces, Differential Operators. Amsterdam - New York - Oxford: North-Holland Publishing Company, 1978. 
Author's address

\section{Arezki Kheloufi}

Bejaia University, Department of Technology, Lab. of Applied Mathematics, 06000 Bejaia, Algeria E-mail address: arezkinet2000@yahoo.fr 